\title{
Experimental Verification of Distributed Parameters on Indian Residential Networks for Power Line Communication
}

\author{
Shashidhar Kasthala ${ }^{\# 1}$, Prasanna Venkatesan GKD *2 \\ ${ }^{\# 1}$ Karpagam Academy of Higher Education, Coimbatore, Tamil Nadu, India \\ ${ }^{1}$ shashi_kb4u@rediffmail.com \\ ${ }^{* 2}$ SNS College of Engineering, Coimbatore, Tamil Nadu, India \\ ${ }^{2}$ prasphd@gmail.com
}

\begin{abstract}
To characterize and model an indoor power line channel, it is of utmost importance to estimate the distributed parameters accurately. These distributed parameters are influenced by several factors that may vary from each location. In this paper, the distributed parameters are estimated on Indian residential networks considering the different aspects of electrical wiring. The results obtained through simulation are compared with other commercially available simulators and are also verified experimentally using a Vector Network Analyser. The attenuation for different wiring configurations is also obtained in the paper.
\end{abstract}

Keyword - Attenuation, Channel transfer function, Distributed parameters, Impedance.

\section{INTRODUCTION}

Communication over power line cables has opened new avenues in home network connectivity by ensuring data rates as high as 200 Mbps. Though PLC has a long way ahead for full scale deployment, researchers are optimistic about the commercial implementation of this technology. The major hurdle in PLC system design is to understand the parameters like noise, multipath and attenuation which make the power line a very unfriendly channel for communication. Unfortunately, these parameters not only vary with time, but also with location and topology of the network thus becoming difficult to characterize and model a power line channel.

The noise in PLC channel is due to the inherent nature of the power line called as background noise and/or because of the very nature of the loads connected to the network called as impulsive noise. The background noise is regarded as stationary noise because of its nominal variation and the impulsive noise can be synchronous or asynchronous to the mains frequency depending on the type of loads connected. These impulsive noises vary with time and as a significant impact on the BER [1].

Similar to wireless channels, the PLC channels also exhibit multipath characteristics (transmission and reflection of signals) due to the varied branch length and different loads connected throughout the power line. This multipath effect degrades the signal transmitted over the power line [2]. Similarly, the attenuation in the power line is due to the distinct power cables used and the variety of loads connected across the network. One predominant factor for attenuation in the power line is due to the reflections of branch terminals [3].

Taking into account the complexity of power line channel, researchers have developed various channel modeling techniques so far which can be classified into top-down or bottom-top approach [4],[5]. The top-down approach of channel modeling is based on the multipath propagation and where as the bottom-top approach is an analytical method developed on the physical and electrical properties extracted from the network. These power line channels can be modeled using any of these approaches either in time domain or in frequency domain. In fact PLC simulators are also commercially available based upon these models [6-8].

However, to characterize a power line channel, it is very important to accurately estimate the distributed parameters of an electrical line. The various factors that influence the channel parameters are Skin effect, proximity effect, type of wiring used, wiring geometry in the cable, coupling effect etc. apart from the weather conditions which is difficult to simulate. One such detailed study is carried out in this paper to estimate the line parameters on Indian residential networks. It is nevertheless to say that the electrical distribution scenario varies from each country and so is the impact on the power line [18].

The subsequent sections of the paper are organized as follows. The section II discuss about the various factors that influence the power line channel model. In section III, the results obtained from the proposed model are compared with the models proposed by other researchers. In section IV, the results are verified experimentally on an electrical wire using a Vector Network Analyzer. In this section, the study is also extended to understand the power line behaviour for different configurations and wiring sizes. In section V, the channel frequency response is estimated on a sample network and the results are compared with the proposed model. 


\section{Distributed PARAMETers for Residential EleCtrical Wiring}

The residential electrical network in India typically contains 3 wires viz. the Live or Phase wire (L), Neutral wire $(\mathrm{N})$ and earth wire (E) as shown in Fig. 1. The phase and neutral wires enter the distribution box connected through the service mains and the earth wire is terminated in the house premises. These wires are very loosely twisted together and connected from the PVC switch boxes to different appliances in the room. These wires are drawn through the PVC conduit pipes along the concrete walls of a room. The conductors used in the residential wiring are usually with a cross-section of either 1.5 sq. $\mathrm{mm}$ or $2.5 \mathrm{sq}$. $\mathrm{mm}$ based on electrical appliances used [11].

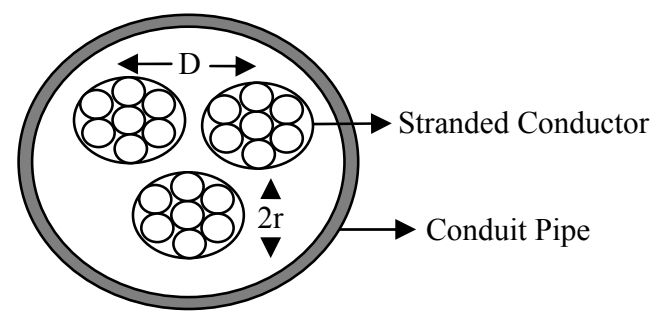

Fig. 1. 3-core residential electrical wiring

\section{A. Resistance}

The Resistance of the single core cable for a two-wire transmission line is given as

$$
R=\frac{1}{\pi r \delta \sigma}\left[\frac{\frac{D}{2 r}}{\sqrt{\left(\frac{D}{2 r}\right)^{2}-1}}\right]
$$

The skin depth $(\delta)$ of the cable is given by

$$
\delta=\frac{1}{\sqrt{\pi f \mu \sigma}}
$$

Where $\mathrm{r}$ is the radius of the conductor, $\mathrm{D}$ is the distance between the conductors, $\sigma$ is the conductivity and $\mu$ is the permeability of the cable. However, if the cable is stranded in nature, the correction factor is given as

$$
X_{R}=\frac{\left[\cos ^{-1}\left(\frac{r_{w}-\delta}{r_{w}}\right) r_{w}^{2}-\left(r_{w}-\delta\right) \sqrt{r_{w}^{2}-\left(r_{w}-\delta\right)^{2}}\right]}{2 r_{w} \delta}
$$

Where $r_{\mathrm{w}}$ is the radius of each strand in the conductor.

The resistance obtained in (1) is for unit length of a single conductor. If a two-wire transmission model is considered, the resistance has to be doubled. For a 3-wire residential network, the presence of earth wire has an influence on the overall resistance due to proximity effect.

\section{B. Inductance}

The inductance of two-wire power line cable includes the self-inductance of each conductor and mutual inductance between them. It is given as

$$
L=\frac{\mu_{r} \mu_{o}}{\pi} \cosh ^{-1}\left(\frac{D}{2 r}\right)+\frac{R}{2 \pi f}
$$

Where, $\mu_{\mathrm{r}}$ is the relative permeability of the conductor.

For a stranded conductor a correction factor should also to be multiplied to the total inductance, but many authors neglect this value [16].

$$
X_{w}=\frac{n_{w} \pi r_{w}^{2}}{\pi r^{2}}
$$

Where $\mathrm{n}_{\mathrm{w}}$ is the number of strands in the conductor

As per [14], the effective resistance of the wire may increase due to skin effect but the inductance of the wire decreases. The correction factor for the skin effect on the inductance is given as

$$
X_{L}=\frac{\delta}{0.135 \times 0.53 \times r_{w}}
$$

The presence of earth wire in the conduit and the geometry of the cables placed in the conduit will affect the overall inductance leading to an additional factor of uncertainty.

\section{Capacitance}

Since the cables in residential electrical networks are placed in conduit pipes, the capacitive coupling effects of the conduit, the geometry of the cable and the cable twist also affect the capacitance of the wiring. As per [15], for a twisted power line, the correction factor for equivalent dielectric constant $\left(\varepsilon_{q}\right)$ is given as 


$$
X_{t}=0.45+\left(\operatorname{atan}(T . \pi . D) \times \frac{180}{\pi}\right)^{2} \times 10^{-3}
$$

Where, $\mathrm{T}$ is the number of twists per meter length of wiring. Though the wires in the residential building are loosely twisted, the impact on the capacitance cannot be neglected due to the smaller cross-section of the conduit. Another aspect that is needed to be taken in to account is the geometry of the cable. The three wires are placed neither equilaterally nor right angled throughout the conduit, but certainly have an impact on the capacitance.

The capacitance between any two conductors is given as

$$
C_{C b l}=\frac{\pi \varepsilon_{o} \varepsilon_{q}}{\ln \left[\left(\frac{D}{2 r}\right)+\sqrt{\left(\frac{D}{2 r}\right)^{2}-1}\right]}
$$

Where $\varepsilon_{\mathrm{q}}$ is the equivalent dielectric constant obtained by

$$
\varepsilon_{q}=1+X_{t}\left(\varepsilon_{r}-1\right)
$$

As per [12], the total capacitance of a cable when placed in a conduit is given as

$$
C=3 \frac{C_{C b l}}{2}+\frac{C_{c n d t}}{2}
$$

Where $\mathrm{C}_{\mathrm{cndt}}$ is the capacitance between the cable and the conduit which is given as

$$
C_{c n d t}=\frac{\pi \varepsilon_{c}}{\ln \left(\frac{b}{r}\right)}
$$

Here, $\varepsilon_{\mathrm{c}}$ is the dielectric permittivity between the cable and conduit and $\mathrm{b}$ is the radius of the conduit.

\section{Conductance}

The conductance of the cable is given by the equation

$$
\mathrm{G}_{\mathrm{cbl}}=2 \pi \mathrm{fC} \tan \delta
$$

The presence of the conduit influences the conductance of the cable and it can be obtained directly from the capacitance in (10).

$$
\mathrm{G}=\frac{\mathrm{G}_{\mathrm{cbl} \cdot \mathrm{C}}}{\mathrm{C}_{\mathrm{cbl}}}
$$

The four intrinsic parameters obtained from (1) to (13) can be used to determine the characteristic impedance (Zo) and the propagation constant $(\gamma)$. The Characteristic impedance is given as:

$$
\mathrm{Z}_{\mathrm{o}}=\sqrt{\frac{(\mathrm{R}+\mathrm{jwL})}{(\mathrm{G}+\mathrm{jwC})}}
$$

and the propagation constant is given by

$$
\gamma=\sqrt{(\mathrm{R}+\mathrm{jwL})(\mathrm{G}+\mathrm{jwC})}
$$

\section{Comparison of Proposed Parameters With OTHER Simulators}

In the last decade, researchers have developed numerous models to characterize and model a power line channel. In each model certain assumptions are made in estimating the distributed parameters. In the subsequent paragraphs of this section, few such model and their assumptions are discussed,

The FTW PLC simulator [6] realized in MATLAB makes use of the technical data published by the cable manufacturer. In the simulation method, the Inductance and Capacitance are frequency independent and the effective Resistance and conductance are given by

$$
\begin{aligned}
& R=R^{\prime} \cdot \sqrt{f} \cdot 10^{-5} \\
& G=G^{\prime} \cdot 2 \pi f \cdot 10^{-14}
\end{aligned}
$$

The channel parameters as proposed by Mlynek in [8] are frequency dependant unlike the FTW PLC simulator. But the distributed parameters are insensitive to the factors mentioned in the previous section except skin effect on the resistance.

Meng has assumed in [12] that the transmission line parameters are based on several factors that influence the R, L, G, C parameters. The effect of strands on resistance, the influence of earth wire and the effect of conduit on the capacitance are considered in estimating these distributed parameters.

In all the above three approaches, the authors have not considered the proximity effect on resistance, the geometry of the cable, twist pitch and the effect of skin depth on the inductance.

In this article, a comparative analysis is carried out on all the above mentioned models. The cable used for the study is the commonly used 2.5 sq.mm cable of size $36 / 0.3 \mathrm{~mm}$. The conductor is an unsheathed class 2 conductor. For the sake of FTW simulator, the capacitance and inductance values of the cable are deliberated as 
$15 \mathrm{pF} / \mathrm{m}$ and $1.08 \mu \mathrm{H} / \mathrm{m}$. These results are compared with the distributed parameter values obtained from the proposed model.

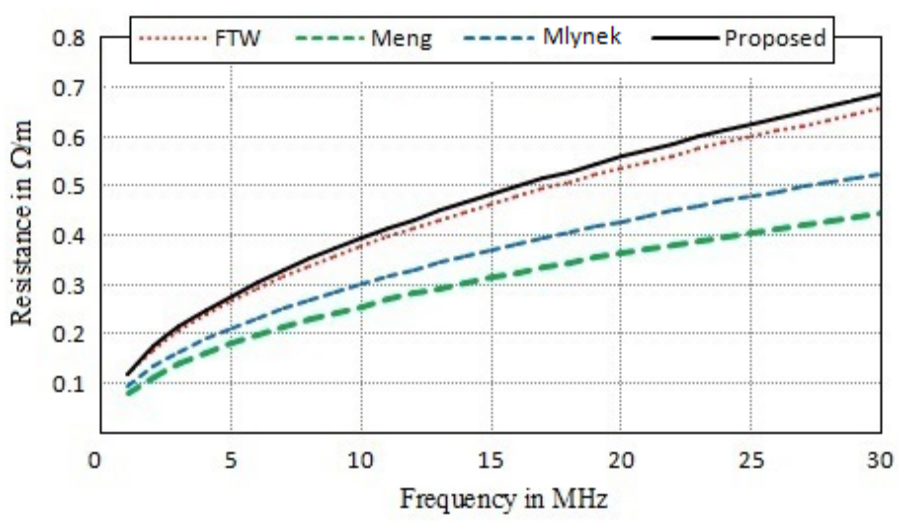

Fig. 2. Comparison of resistance obtained from different simulators

The distributed parameters R, L, G and C obtained from the various simulation methods are compared with the proposed method and are shown in Fig. 2, Fig. 3, Fig. 4 and Fig. 5 respectively. It can be depicted from the figures that the distributed parameters are dependent upon the nature of material used; spacing between the live and neutral wire, frequency of operation and physical properties of the wiring.

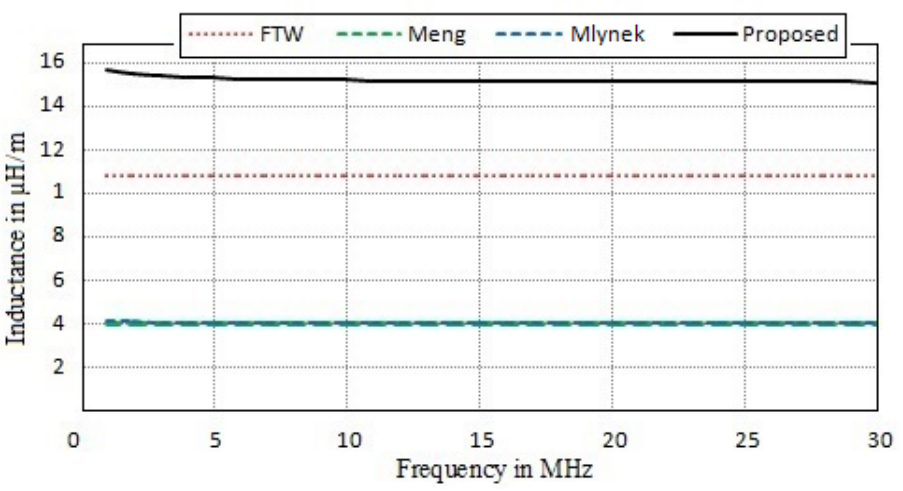

Fig. 3. Comparison of inductance obtained from different simulators

The increase in the resistance can be attributed to the correction factor due to stranded conductor and due to the proximity effect of the earth wire. Similarly, the significant difference in inductance is observed when compared to other methods due to the multiplying factor considered for the geometry of the cable laid in the conduit. This is in addition to the skin effect.

Similarly, the variation in the capacitance and conductance by the proposed parameters is due to the multiplication factor from the geometry of the cable and the number of twists of the wiring placed in the conduit pipe. The capacitance between the wires and the conduit pipe also has a significant influence on the capacitance. In the proposed method, these values are found to be different for as the cross-section of wiring is changed, but in FTW PLC simulator L and C are the same irrespective of cross-section of the wire.

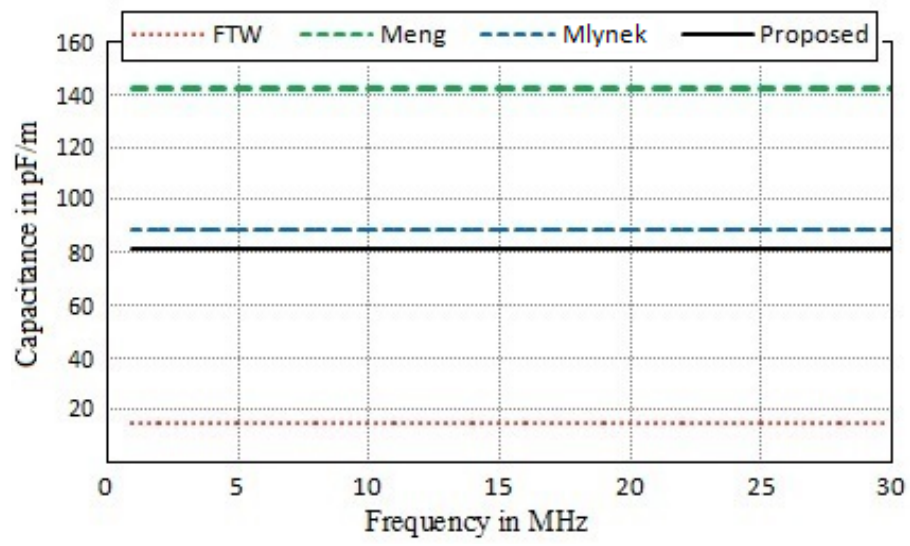

Fig. 4. Comparison of capacitance obtained from different simulators 


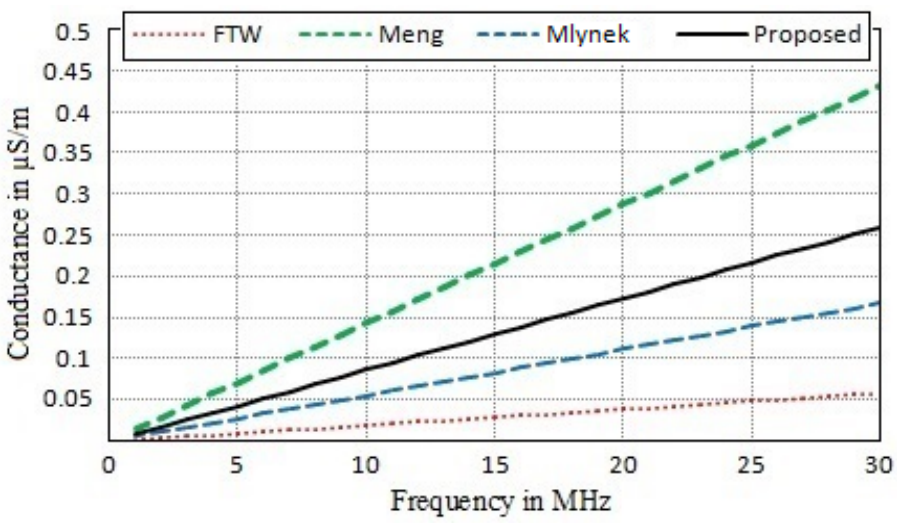

Fig. 5. Comparison of conductance obtained from different simulators

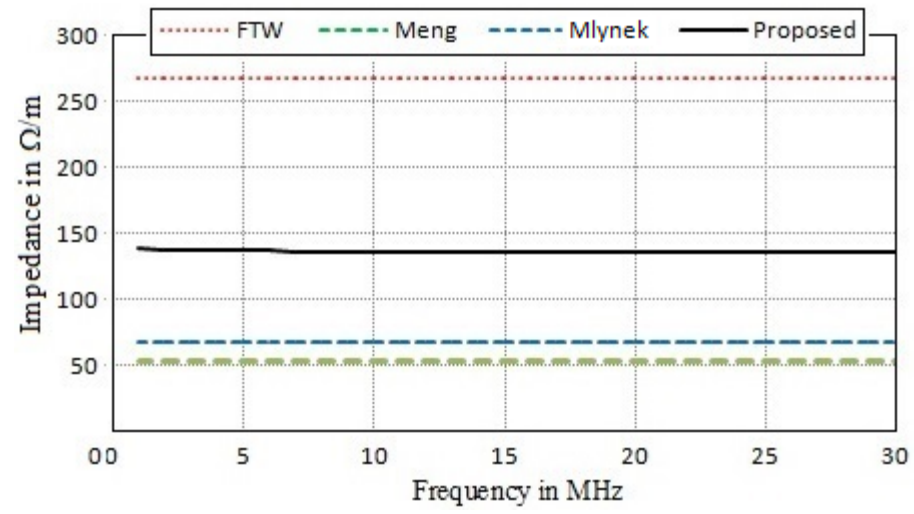

Fig. 6. Comparison of Impedance obtained from different simulators

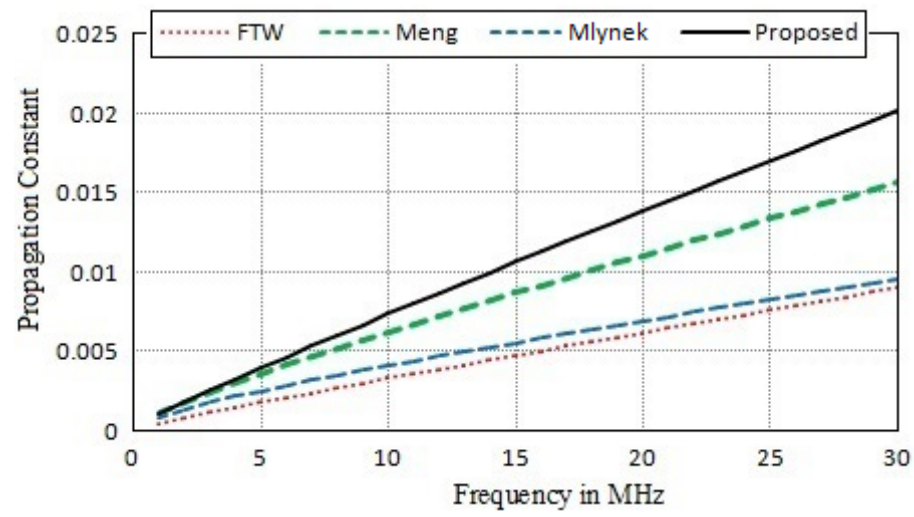

Fig. 7. Comparison of Propagation Constant obtained from different simulators

From the distributed parameters obtained, the intrinsic parameters viz. impedance and propagation constant can be estimated using (18) and (19). As shown in Fig. 6, the impedance obtained from the proposed method is almost two times higher than the models proposed by Mlynek and Meng but significantly less than the one proposed in FTW PLC simulator.

\section{EXPERIMENTAL VERIFICATION OF PARAMETERS}

A typical Indian residential wiring of $3 \times 2.5$ sq.mm unsheathed flexible PVC cable placed in a conduit is considered for experimental verification of the parameters. The measurements are carried out using the R\&S ZVL Network Analyser.

Using the Network Analyzer, the input impedance of open circuit and short circuit ended cables is obtained and from the transmission line theory, the impedance of the power line can be obtained as

$$
Z=\sqrt{Z_{o c} Z_{s c}}
$$

Where, $Z_{\mathrm{oc}} Z_{\mathrm{sc}}$ is the input impedances of open circuited and short circuited cables respectively. 


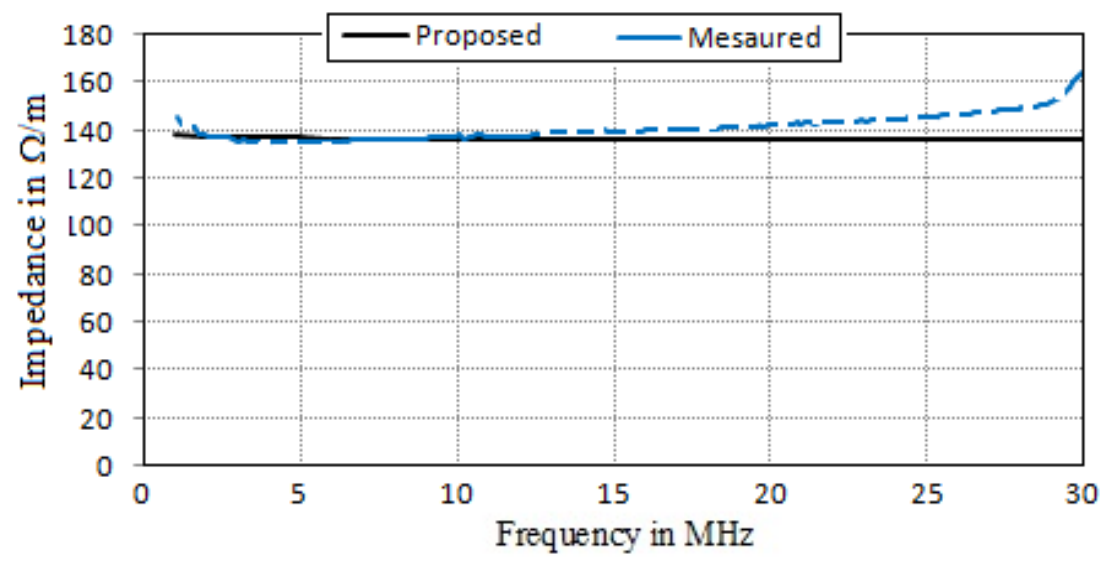

Fig. 8. Impedance measured Vs Impedance simulated

The impedance obtained experimentally is compared with the simulated value from the proposed method and the same is shown in Fig. 8. It can be observed that the impedance obtained from the proposed distributed parameters is almost similar to the impedance achieved through measurements.

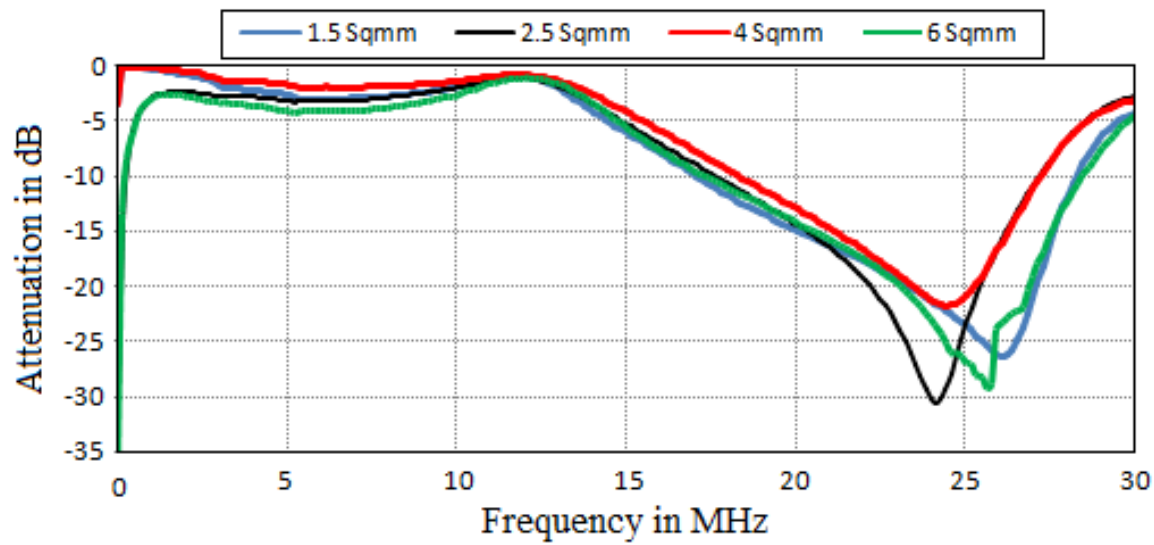

Fig. 9. Attenuation for different cable dimensions

The wiring in the residences need not be of uniform cross-section throughout. It differs with the appliances connected to it, so as to carry the load current without exceeding the thermal limits of the insulation. For example, 1.5 sq.mm wiring can be used for a simple lighting circuit but for a higher rating device like oven or refrigerator, a 2.5 sq.mm wiring is used. Fig. 9 represents the transfer function measured in terms of attenuation for different cable dimensions commonly used in Indian residences. It can be observed from the figure that, there is a little change in the attenuation among different cable dimensions.

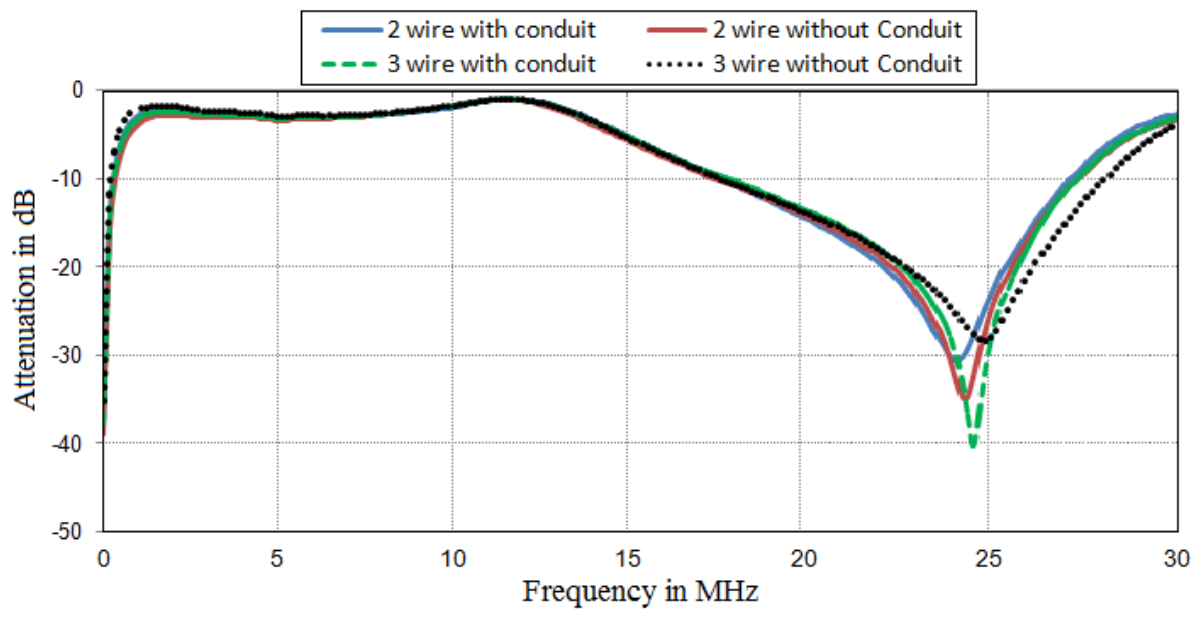

Fig. 10. Attenuation for different Cable Configurations 
Typically, the residential electrical wiring in India contains of three wires viz., live, earth and neutral, but in such locations of the residence where class II electronics devices (which operate on low current) are to be connected, earth wire may not be used. In that case Fig. 10 shows the attenuation of two wiring and three wiring systems placed in a conduit pipe and placed without a conduit pipe. It can be perceived from the figure that the wiring placed in the conduit pipe has more attenuation then the one without the conduit pipe.

\section{Channel Frequency Response}

A sample single branch network as shown in Fig. 11 is examined in the article to verify the channel transfer function obtained from the proposed distributed parameters. The transfer function in terms of attenuation is measured from the $S_{21}$ parameters of Network Analyser. In this exercise, the frequency response up to $30 \mathrm{MHz}$ is only measured for the sake of simplicity.

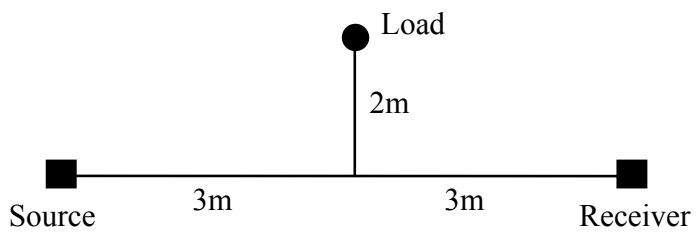

Fig. 11. Sample network with single branch

The channel frequency response of a single branch network is simulated using the transmission line theory approach as in [17]. The obtained Amplitude response with the proposed distributed parameters is compared with the measured transfer function $\mathrm{S}_{21}$ of the analyser. The same is represented in Fig. 12.

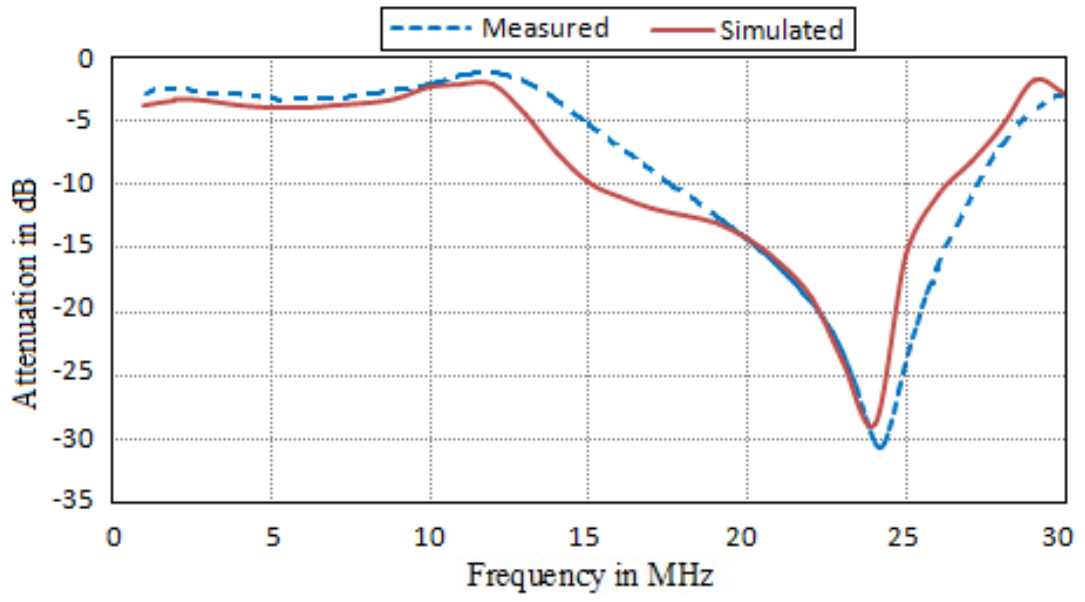

Fig. 12. Channel Transfer function of a single branch circuit

The position of peaks and notches of the derived amplitude response almost match with the measured values, by which it can be deduced that an extensive analysis need to be carried out in understanding the various factors influencing the parameters.

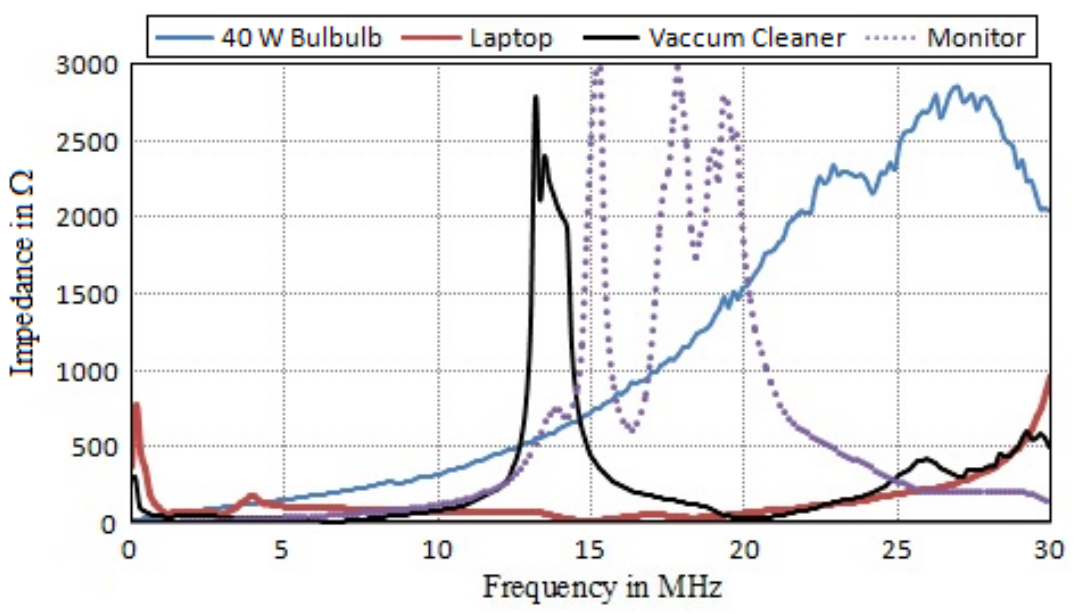

Fig. 13. Impedances of few domestic appliances 
For further study of the channel transfer function, the impedance of few typically used electrical appliances is measured and is shown in Fig. 13. The loads considered in this article are 40 watts incandescent bulb, a laptop, 1200 watts Vacuum Cleaner and LED TV Monitor. It can be noticed that, at a particular frequency there is sharp change in the impedances for appliances.

The impedance of these loads is obtained in two stages. First, the impedance of the line $\left(Z_{\text {Line }}\right)$ is measured without any load connected to the network and then the impedance of line is measured with different loads $\left(\mathrm{Z}_{\text {Tot }}\right)$ connected to the network. Since the line and the load are in parallel to each other, the total impedance of the line will be parallel sum of line impedance and the load impedance. Thus the load impedance $\left(\mathrm{Z}_{\text {Load }}\right)$ can be obtained by

$$
Z_{\text {load }}=\frac{Z_{\text {Line }} \times Z_{\text {Tot }}}{\left|Z_{\text {Line }}-Z_{\text {Tot }}\right|}
$$

\section{CONCLUSION}

The distributed parameters are evaluated on Indian residential networks by considering several factors that influence the power line. The obtained results from the proposed model are compared in detail with the available simulators. These values are also verified experimentally. In addition to the analysis made on the distributed parameters, the input impedance may also have an impact on the results obtained. Apart from the physical characteristics and material used for the wiring, the environment and the ambient conditions will also impact the distributed parameters. The channel transfer function is simulated for a sample network on the proposed parameters and verified experimentally. For further study, the behaviour of typical domestic appliances is also evaluated.

\section{REFERENCES}

[1] Luca Di Bert, Peter Caldera, David Schwingshackl and Andrea M.Tonello, "On Noise Modeling for Power Line Communications," IEEE International Symposium on Power Line Communications \& Applications, pp. 283-288, 2011.

[2] Justinan Anatory, Nelson Theethayi, Rajeev Thottappillil," Effects of Multipath on OFDM Systems for Indoor Broadband Power-Line Communication Networks," IEEE Transactions on Power Delivery. vol. 24(3), pp.1190-1197, 2009.

[3] T.Maenou and M.katayama, "Study on Signal Attenuation Characteristics in Power Line Communications," IEEE International Symposium on Power Line Communications \& Applications, pp. 217-221, 2006.

[4] Haroldo Zattar, Lucas Salek and Gilberto Carrijo, "An Evaluation of Power line Communication Channel Modeling for Indoor Environment Application," 2nd national conference on Telecommunications (CONATEL), 2011.

[5] Wenfei Zhu, Xu Zhu, Enggee Lim and Yi Huang, "State-of-art Power Line Communication Channel Modeling," Procedia Computer Science, vol. 17, pp. 563-570, 2013.

[6] G.Marrocco, D.Statovci, and Martin Wolkerstorfer, FTW PLC Simulator [computer software]. Wien, Austria, 2014.

[7] F.Canete, J.Cortes, L.Diez and J.Entrambasaguas, "A Channel Model Proposal for Indoor Power Line Communications," IEEE Communications Magazine, vol.49 (12), pp.166-174, 2011.

[8] P.Mlynek, J.Misurec, M.Koutny, R.Fujdiak and T.Jedlicka, "Analysis and Experimental Evaluation of Power Line Transmission Parameters for Power Line Communication” Measurement Science Review, vol. 15(2), pp.64-71, 2015

[9] G.T.Andreou and D.P.labridis, "Electrical Parameters of Low-Voltage Power Distribution cables Used for Power-Line Communications," IEEE Transactions on Power delivery vol. 22(2), pp.879-886, 2007.

[10] P.Wagenaars, P.A.A.F. Wouters, P.C.J.M van der Wielen, E.F.Steennis, "Measurement of Transmission Line Parameters of Three Core Power Cables with Common Earth Screen," IET Science, Measurement and Technology, vol. 4(3), pp.146-155, 2010.

[11] Xavier Carcelle, "Power Line Communications in Practice," Artech House, 2009.

[12] H.Meng, S.Chen, Y.L.Guan, C.L.Law, P.L.So, E.Gunawan, and T.T.Lie, "Modeling of Transfer Characteristics for the Broadband Power Line Communication Channel," IEEE Transactions on Power Delivery, Vol. 19(3), pp. 1057-1064, 2004.

[13] T.S.pang, P.L.So and K.Y.Lee, "Common-Mode Current propagation in Power Line Communication Networks using MultiConductor Transmission Line Theory," IEEE International Symposium on Power Line Communications \& Applications, pp. 517-552, 2007.

[14] S.Kim and D.P.Neikirk, "Compact Equivalent Circuit Model for Skin Effect," IEEE-MTTS International Microwave symposium Digest, 1996

[15] T Kien Truong, “Twisted Pair Transmission Line Distributed Parameters," 1969.

[16] Despina Anastasiadou and Theodore Antonakopoulos, "An Experimental Setup for Characterizing the Residential Power Grid variable behavior," International Symposium on Power Line Communications and its Applications, 2002.

[17] Shashidhar Kasthala and GKD Prasanna Venkatesan, "Estimation of MIMO Power Line Communication Channel Capacity using Multi-Conductor Transmission Line Theory: 2nd International Conference on Applied and Theoretical Computing and Communication Technology, 2016.

[18] “General Specifications for Electrical Works, part -1, Internal," CPWD, Government of India, 2005.

\section{AUTHOR PROFILE}

Shashidhar Kasthala is working as Assistant Professor in Indian Naval Academy, Ezhimala and currently pursuing his Ph.D from Karpagam Academy of Higher Education, Coimbatore, India. His areas of research interest are Power Quality and Power Line Communication.

Dr. G.K.D Prasanna Venkatesan has received his Ph.D from Anna University in 2007. He is presently working as professor and Head, Research \& Development Cell, SNS College of Engineering, Coimbatore, India. His areas of research interest are Internet of Things, Wireless Mobile Communication, Computer Networks and Information Security System. 